\title{
Acupuncture in the Treatment of Chronic Prostatitis/Chronic Pelvic Pain Syndrome: A Brief Review
}

\begin{abstract}
Introduction/Aim: Chronic prostatitis/chronic pelvic pain syndrome (CP/CPPS) affects many adult men worldwide. It has been almost a decade since the introduction of acupuncture on $\mathrm{CP} / \mathrm{CPPS}$ treatment. Since then, a number of studies have been performed. The aim of the study was to assess the effects and safety of the use of acupuncture for CP/CPPS. Materials and Methods: A systematic search was performed in electronic libraries for clinical trials, experimental studies, and systematic reviews on the topic using the terms: "chronic prostatitis," "chronic pelvic pain syndrome," "acupuncture" combined with the keywords: "treatment," "efficacy," and "safety," in various combinations. In order to provide accurate conclusions, we evaluated only randomized studies focused on the effects and safety data of acupuncture in the treatment of CP/CPPS-related symptoms. Only trials performed in patients with confirmed CP/CPPS randomized with adequate methods and providing clear outcome reports were finally evaluated. Only full-text available papers written in the English language were considered. There was no restriction on publication date. Results: According to our research, 40 papers examining the role of the acupuncture in the treatment of $\mathrm{CP} / \mathrm{CPPS}$ exist. Only 8 out of 40 fulfilled the above-mentioned criteria. Overall, evidence supports acupuncture as an effective treatment for CP/CPPS-induced symptoms, particularly in relieving pain. Regarding long-term responses without additional treatment, the examined studies provide inconsistent information. Moreover, evidence regarding urination problems is limited. Conclusion: Available data suggest that acupuncture treatment is able to decrease CP/CPPS related pain. Since it was associated with rare and slightly adverse events, it could be considered as a safe complementary therapeutic option for men with CP/CPPS.
\end{abstract}

Keywords: Chronic prostatitis, acupuncture, chronic pelvic pain syndrome

\section{Introduction/Aim}

Acupuncture is a form of alternative medicine and a key component of traditional Chinese medicine in which thin needles are inserted into specific points on the body. ${ }^{[1]}$ It is mainly used to cure chronic back and neck pain, though it is believed that it can also be used for a wide range of other conditions. ${ }^{[2]}$ It is almost a decade that acupuncture has been used somehow systematically in Western countries for the treatment of symptoms in chronic prostatitis/chronic pelvic pain syndrome (CP/CPPS). The aim of the study was to assess the effects and safety of the use of acupuncture for CP/CPPS.

\section{Materials and Methods}

A systematic search was performed in electronic libraries for clinical trials,

This is an open access journal, and articles are distributed under the terms of the Creative Commons Attribution-NonCommercial-ShareAlike 4.0 License, which allows others to remix, tweak, and build upon the work non-commercially, as long as appropriate credit is given and the new creations are licensed under the identical terms.

For reprints contact:WKHLRPMedknow_reprints@wolterskluwer.com experimental studies, and systematic reviews on the topic using the terms: "chronic prostatitis," "chronic pelvic pain syndrome," "acupuncture" combined with the keywords: "treatment," "efficacy," "effects," and "safety," in various combinations. Bibliographic information in the selected publications was checked for relevant publications not included in the initial search. In order to provide accurate conclusions, we evaluated only randomized studies focused on the effects and safety data of acupuncture in the treatment of CP/CPPS-related symptoms. Only trials performed in patients with confirmed CP/CPPS randomized with adequate methods and providing clear outcome reports were finally evaluated. Review articles and experimental animal trials and those including participants with acute bacterial prostatitis, benign prostate enlargement, prostate cancer, or other prostate diseases were excluded.

How to cite this article: Stamatiou K, Samara E, Kefalas K, Perletti G. Acupuncture in the treatment of chronic prostatitis/chronic pelvic pain syndrome: A brief review. Hellenic Urol 2021;32:117-20.

\section{Konstantinos \\ Stamatiou, Evangelia Samara1, Konstantinos Kefalas, Gianpaolo Perletti ${ }^{2,3}$}

Department of Urology, Tzaneion General Prefecture Hospital of Piraeus,

${ }^{1}$ Department of Chronic Pain Care, Tzaneion General Prefecture Hospital of Piraeus, Greece, ${ }^{2}$ Department of Biotechnology and Life Sciences, University of Insubria, Varese, Italy, ${ }^{3}$ Department of Human Structure and Repair, Ghent University, Ghent, Belgium

Submitted: 23-Dec-2020

Revised: 24-Dec-2020

Accepted: 24-Dec-2020

Published: 22-Feb-2021

Address for correspondence: Dr. Konstantinos Stamatiou, 2 Salepoula Street, 18536 Piraeus, Greece. E-mail:stamatiouk@gmail.com

Access this article online

Website: www. hellenicurologyjournal.com

DOI: 10.4103/HUAJ.HUAJ_17_20 Quick Response Code:

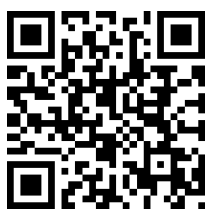


Only full-text available papers written in the English language were considered. There was no restriction on the publication date.

\section{Results}

According to our research, 40 papers examining the role of the acupuncture in the treatment of $\mathrm{CP} / \mathrm{CPPS}$ exist. Only 8 out of 40 fulfilled the above-mentioned criteria.

Küçük et al. compared the electro-acupuncture treatment (sacral nerve stimulation, twice a week for 7 weeks) to the medical treatment (levofloxacin $500 \mathrm{mg}$ daily and ibuprofen $200 \mathrm{mg}$ twice a day for 6 weeks). The mean follow-up was 28 weeks from the baseline (range, 20-43 weeks). In acupuncture group $(n=26)$, reduction of pain, urinary symptoms, quality of life, and total National Institutes of Health Chronic Prostatitis Symptom Index score (NIH-CPSI) were higher compared with the medical group $(n=28) \cdot{ }^{[3]}$
Capodice et al. provided full body and auricular acupuncture treatment twice weekly for 6 weeks to 10 men diagnosed with $\mathrm{CP} / \mathrm{CPPS}$ for 6 months. In all cases, symptoms were refractory to at least 1 conventional therapy (antibiotics, anti-inflammatory agents, 5-alpha reductase inhibitors, and alpha-1 blockers) and scoring $>4$ on the pain subset of the NIH-CPSI. After 3 and 6 weeks from baseline, significant changes in total NIH-CPSI and QOL scores were noted. They remained significant after an additional 6 weeks of follow-up. ${ }^{[4]}$

Zhou et al. compared long-needle acupuncture (LA) and traditional acupuncture (TA) in a small single-blind study. Seventy-seven patients received six sessions of acupuncture for 2 weeks and a follow-up was scheduled at week 24. The primary outcome was measured by the total NIH-CPSI. Four domains of the NIH-CPSI (urination, pain or discomfort, effects of symptoms, and quality of

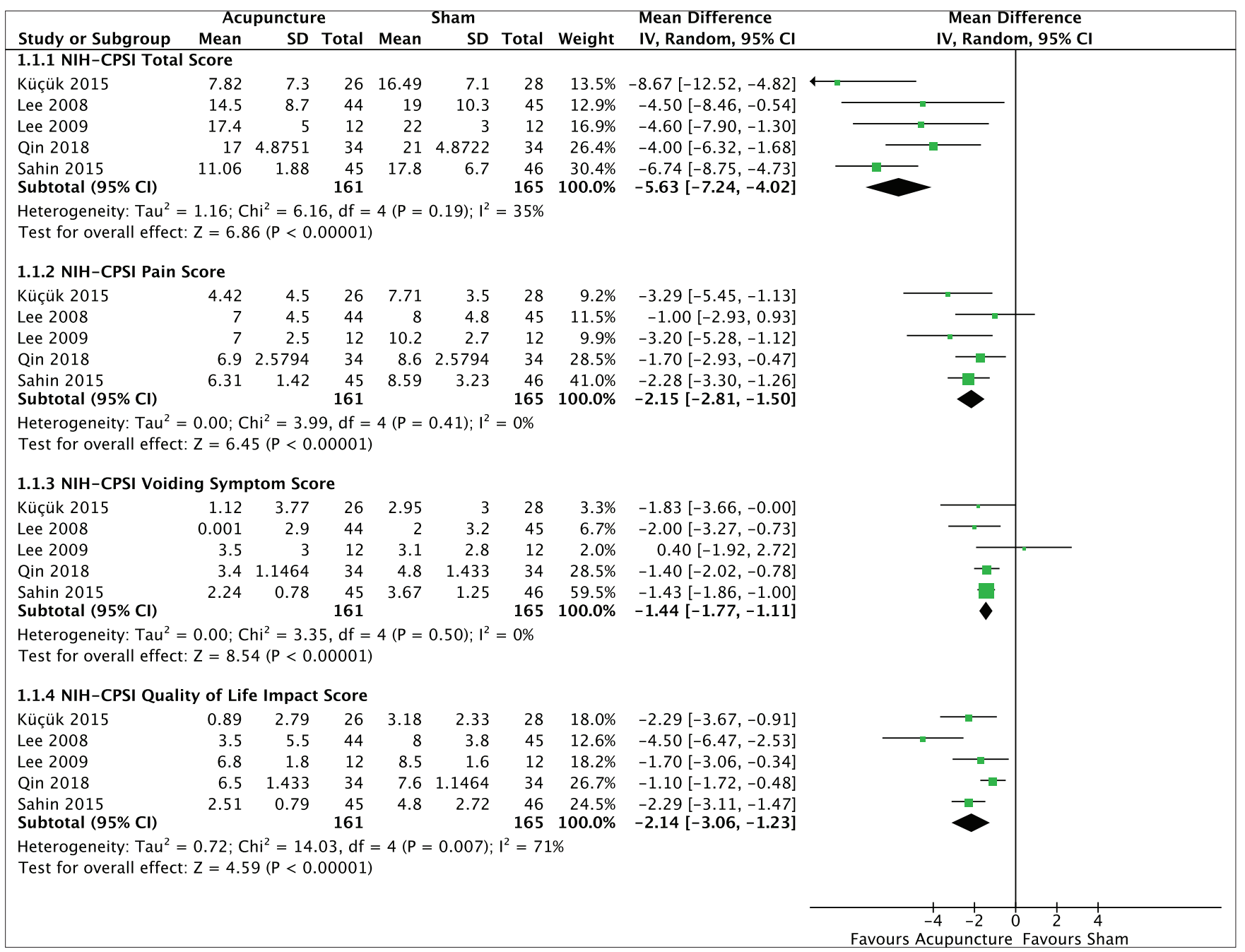

Figure 1. Pooled summary of 5 out of total 8 studies included in this review, comparing the effect of acupuncture versus sham acupuncture (with or without adjuvant medical or physical therapy) in CP/CPPS patients. Forest plots indicate the mean differences of NIH-CPSI total scores, and pain, voiding and QoL NIH-CPSI subscores, assessed at the end of treatment (range: 6 to 10 weeks). Only randomized controlled trials were included. Data to the left of the vertical no-effect line of forest plots represent a favorable effect of acupuncture vs. sham acupuncture. Diamonds represent overall effect sizes extending to the limits of the $95 \%$ confidence intervals of mean differences. The significance of the pooled effect sizes (Z statistics) and heterogeneity data (I^2, Chi-square, $\mathrm{P}$ values) are shown. Inverse variance statistics, random effects analysis model. RevMan 5.3. 
life) and the clinical efficacy score served as the secondary outcome. The total NIH-CPSI score at weeks 2 and 24 was significantly improved in the LA group compared with the TA group. LA significantly improved urination, pain or discomfort, the effects of symptoms, and the quality of life at weeks 2 and $24 .^{[5]}$

Qin et al. performed a randomized, placebo-controlled (sham acupuncture) trial with 8 weeks of treatment followed by 24 weeks of follow-up. The primary outcome was the change in the NIH-CPSI total score from baseline to week 8. The secondary outcomes were the NIH-CPSI subscale scores, pain severity, the International Prostate Symptom Score (IPSS), the global response rate, and satisfaction assessment. According to the authors, the reduction in the NIH-CPSI total score differed significantly between the two groups at weeks 8,20, and 32. All differences were greater than the 4-point minimal clinically important difference. For all other secondary outcomes, the acupuncture group was statistically better than the sham acupuncture group. ${ }^{[6]}$ Lee et al. randomized CP/CPPS patients who had a NIH-CPSI total score $\geq 15$ on the and symptoms for at least 3 of the preceding 6 months to acupuncture or sham acupuncture. Treatment consisted of twice weekly 30 -min sessions for 10 weeks (20 sessions total) without needle stimulation, herbs, or adjuvants. The primary response criterion was a 6-point decrease from baseline to week 10 in NIH-CPSI total score (range 0-43). The investigators reported that $32(73 \%)$ of 44 participants responded in the acupuncture group compared with 21 (47\%) of 45 sham group. However, long-term responses 24 weeks after completing therapy without additional treatment occurred in $14(32 \%)$ of 44 acupuncture group participants and in $6(13 \%)$ of 45 sham group. ${ }^{[7]}$

A similar, quite larger study by Sahin et al. found that the $92 \%$ of the participants of the real acupuncture group reported $>50 \%$ decrease in total NIH-CPSI score from baseline compared to the $48 \%$ of sham participants, at 8 weeks after the end of the therapy. Both groups experienced significant decrease in CPSI subscores throughout the whole follow-up period; however, the decline remained significantly greater in the active acupuncture group as compared with the sham group. ${ }^{[8]}$

Lee and Lee randomized 39 men to three treatment groups: group 1: advice and exercise (A\&E) plus 12 sessions of electroacupuncture (EA), Group 2, A\&E plus 12 sessions of sham EA (SEA), and Group 3, A\&E alone for 6 weeks. A total of six acupuncture points was used to stimulate the sacral nerve and release the piriformis muscle using an electrical pulse generator. The symptoms related to $\mathrm{CP} /$ CPPS were assessed using the NIH-CPSI, while the degree of inflammation was calculated with prostaglandin E2 and beta-endorphin levels in postmassage urine samples. According to these authors, at 6 weeks, the NIH-CPSI total score had decreased significantly in the EA group compared to the SEA and A and E groups. On a subscale analysis of the NIH-CPSI, the EA group showed significant decreases in pain-related symptoms compared with the SEA and A and E groups. All EA participants $(100 \%)$ experienced at least a 6-point decrease in the NIH-CPSI total score compared with $16.7 \%$ of SEA participants and $25 \%$ of A and $\mathrm{E}$ participants. Of note, the mean prostaglandin E2 level in the postmassage urine samples had significantly decreased in the EA group $(P=0.023)$. In contrast, it had increased in the other two groups. ${ }^{[9]}$

Seong et al. performed a retrospective study on patients treated with electro pharmaco-acupuncture with either $1 \mathrm{ml}$ of Hwanglyunhaedok or saline at CV1. Treatment was applied twice a week every third day for 4 weeks. After treatment, the total IPSS and NIH-CPSI scores were significantly reduced in both groups. Pain domain scores in both groups showed significant decrease $(P<0.01)$. However, urination scores reduced significantly only in HP group ${ }^{[10]}$ None of the above-mentioned studies reported serious adverse events.

\section{Discussion}

$\mathrm{CP} / \mathrm{CPPS}$ is one of the most common chronic inflammatory diseases in adult men affecting almost $15 \%$ of adult men worldwide. ${ }^{[1]}$ The cause remains practically unknown. Several factors have been associated with its causation such as defective urothelial integrity and function, cryptic infections, autoimmunity, endocrine imbalances, pelvic floor muscle spasm, peripheral and central sensitization, and psychosocial conditions. ${ }^{[12]}$ Various interactions between the above may explain CP/CPPS pathogenesis. One theory proposes that contraction of the pelvic floor muscles, leads to the formation of trigger points and pain. The last results in anxiety and thus worsening of the condition. ${ }^{[13]}$ Another proposal is the interplay between psychological factors and dysfunction in the immune, neurological, and endocrine systems. ${ }^{[14]}$

Available therapeutic options for $\mathrm{CP} / \mathrm{CPPS}$ are far from satisfactory for either physicians or patients. The main reason for the lack of effective and uniform therapies is that the etiology of CP/CPPS still remains unknown. A variety of treatments have been used to relieve related symptoms. Treatments can include one or more of these: Antibiotics, anti-inflammatory or muscle-relaxing medicines, alpha-blockers, baths, prostate massage, dietary changes, biofeedback, surgery, other medicines, or herbal treatments. ${ }^{[15]}$ It has been almost a decade since the introduction of acupuncture on CP/CPPS treatment. Since then, a number of studies have been performed. Many of them suggest that acupuncture works particularly well on CP/CPPS associated chronic pain and many researchers state that acupuncture appears to be a reasonable option for people with chronic pain to consider.

Acupuncture is a branch of traditional Chinese medicine. According to Chinese theories, it releases or redirects the body's natural energy trough invigoration of certain points 
by applying needles, heat, and pressure. However, the actual mechanism of action is unknown.

As shown in this brief review, there are few well-randomized, placebo-controlled studies providing clear outcome reports. Almost all these studies are small. Moreover, in all examined studies, the primary outcome assessed was the difference in total NIH-CPSI score between baseline and study completion [Figure 1]. Two studies additionally examined was the difference in total IPSS score between baseline and study completion. Almost all these trials did not provide the variety of decreasing scores but rather the data on baseline and endpoints. Only one study evaluated biological parameters (prostaglandin E2 and beta-endorphin levels). Overall, evidence supports acupuncture as an effective treatment for CP/CPPS-induced symptoms, particularly in relieving pain. Regarding long-term responses without additional treatment, the examined studies provide inconsistent information, and for this reason, no safe conclusions on its potential impact on quality of life and modulation of inflammation cannot be retrieved. Moreover, evidence regarding urination problems is limited.

\section{Conclusion}

Available data suggest that acupuncture treatment is able to decrease $\mathrm{CP} / \mathrm{CPPS}$-related symptoms (Figure 1), since it was associated with rare and mild adverse events it could be considered as a safe complementary therapeutic option for men with $\mathrm{CP} / \mathrm{CPPS}$.

\section{Financial support and sponsorship}

Nil.

\section{Conflicts of interest}

There are no conflicts of interest.

\section{References}

1. Adams D, Cheng F, Jou H, Aung S, Yasui Y, Vohra S. The safety of pediatric acupuncture: A systematic review. Pediatrics 2011;128:e1575-87.

2. Ernst E, Lee MS, Choi TY. Acupuncture: Does it alleviate pain and are there serious risks? A review of reviews. Pain 2011;152:755-64.

3. Küçük EV, Suçeken FY, Bindayı A, Boylu U, Onol FF, Gümüş E. Effectiveness of acupuncture on chronic prostatitis-chronic pelvic pain syndrome category IIIB patients: A prospective, randomized, nonblinded, clinical trial. Urology 2015;85:636-40.

4. Capodice JL, Jin Z, Bemis DL, Samadi D, Stone BA, Kapan S, et al. A pilot study on acupuncture for lower urinary tract symptoms related to chronic prostatitis/chronic pelvic pain. Chin Med 2007;2:1.

5. Zhou M, Yang M, Chen L, Yu C, Zhang W, Ji J, et al. The effectiveness of long-needle acupuncture at acupoints BL30 and BL35 for CP/CPPS: A randomized controlled pilot study. BMC Complement Altern Med 2017;17:263.

6. Qin Z, Zang Z, Zhou K, Wu J, Zhou J, Kwong JSW, et al. Acupuncture for chronic prostatitis/chronic pelvic pain syndrome: A randomized, sham acupuncture controlled trial. J Urol 2018;200:815-22.

7. Lee SW, Liong ML, Yuen KH, Leong WS, Chee C, Cheah PY, et al. Acupuncture versus sham acupuncture for chronic prostatitis/chronic pelvic pain. Am J Med 2008;121:79.e1-7.

8. Sahin S, Bicer M, Eren GA, Tas S, Tugcu V, Tasci AI, et al. Acupuncture relieves symptoms in chronic prostatitis/chronic pelvic pain syndrome: A randomized, sham-controlled trial. Prostate Cancer Prostatic Dis 2015;18:249-54.

9. Lee $\mathrm{SH}$, Lee BC. Electroacupuncture relieves pain in men with chronic prostatitis/chronic pelvic pain syndrome: Three-arm randomized trial. Urology 2009;73:1036-41.

10. Seong KM, Jang G, Kim DW, Kim S, Song BK. Hwanglyunhaedok pharmacopuncture versus saline pharmacopuncture on chronic nonbacterial prostatitis/chronic pelvic pain syndrome. J Acupunct Meridian Stud 2017;10:245-51.

11. Stamatiou K, Avakian R, Zioutos K, Fokas K, Kefalas K, Zavradinos D, et al. Chronic prostatic infection: Epidemiology and clinical characteristics. Hellenic Urol 2019;31:21-27.

12. Bharucha AE, Lee TH. Anorectal and pelvic pain. Mayo Clin Proc 2016;91:1471-86.

13. Anderson RU, Wise D, Nathanson BH. Chronic prostatitis and/ or chronic pelvic pain as a psychoneuromuscular disorder-a meta-analysis. Urology 2018;120:23-9.

14. Pontari MA. Etiology of chronic prostatitis/chronic pelvic pain syndrome: Psychoimmunoneurendocrine dysfunction (PINE syndrome) or just a really bad infection? World J Urol 2013;31:725-32.

15. Doiron RC, Shoskes DA, Nickel JC. Male CP/CPPS: Where do we stand? World J Urol 2019;37:1015-22. 